\title{
Hybrid Micromachining of Transparent Sapphire Substrate by Pulsed Green Laser Irradiation
}

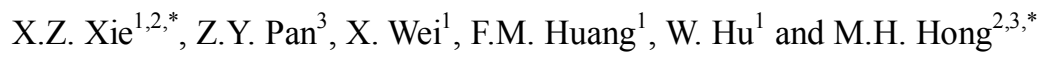 \\ 1- Faculty of Electromechanical Engineering, Guangdong University of Technology, China 510006 \\ 2- Department of Electrical and Computer Engineering, National University of Singapore, \\ Singapore 117576 \\ 3- Data Storage Institute, DSI Building, Singapore 117608 \\ *Corresponding Author: xiaozhuxie@gdut.edu.cn andelehmh@nus.edu.sg
}

\begin{abstract}
Sapphire is an important material for LED substrates and optical windows. Technical challenges of green laser micromachining sapphire include low absorption coefficient and easily to crack as sapphire is transparent from UV to IR light. In this paper, we develop a hybrid laser micromachining technique with the green laser irradiation to enhance the energy absorption to avoid micro-cracks. Firstly, the surface is grooved at a low laser fluence but a higher overlap ratio. At this stage, the surface structure is modified from crystallite to amorphous state, so that its green light absorption increases greatly. Secondly, a subsequent high laser fluence is applied at the same position, and a higher groove depth and narrower groove width can be achieved. Effects of laser fluence on groove depth and width are analyzed. In order to reduce redeposited debris and microcracks, a hybrid laser micromachining at a low laser fluence and multiple scans is conducted. Results indicate that the ablation quality by the hybrid laser micromachining is much better than the direct laser micromachining.

DOI:10.2961/jlmn.2011.03.0007
\end{abstract}

Keywords: Hybrid laser micromachining, transparent sapphire, laser ablation

\section{Introduction}

Transparent \& hard materials, such as sapphire and glass, have been widely used in microelectronics to manufacture devices, operating in the wide temperature and pressure ranges because of its excellent thermal, optical and mechanical properties [1-3]. Due to its brittleness, high hardness, and electrical insulation, the electrical discharge machining (EDM) cannot be used, and the mechanical machining or grinding approaches often have technical challenges of low efficiency and easily to create cracks $[4,5]$. The matter is also strongly resistant to wet and dry chemical etching because of its unique physical properties [6, 7]. Laser micromachining, being a non-contact process, without the problems of mechanical damage and tool wear, is a suitable method for precision machining of sapphire [8]. Direct laser ablation of sapphire has been exploited by nanosecond, picosecond and femtosecond lasers [9-11]. Shorter wavelength lasers are also used in the micromachining of sapphire, including UV Q-switched DPSS (diode pumped solid state) lasers at wavelengths of 355, 266 and $213 \mathrm{~nm}$ and Copper Vapor Laser (CVL) at wavelengths of 255 and $289 \mathrm{~nm}$ [12-14]. Though femtosecond and UV laser ablation of sapphire can achieve high processing quality, high operating cost and low ablation rate limit its industrial applications. The Q-switched pulsed green laser has the merits of high peak power and pulse repetition rate, narrow pulse duration and low operation cost in comparison to UV and ultra-short lasers. Recently, several research groups investigate the pulsed green laser ablation of sapphire [15]. Conventional problems of the laser micromachining sapphire with nanosecond green laser irradiation include low absorption coefficient and easily to crack as sapphire is transparent to the laser wavelength of $532 \mathrm{~nm}$. If the micromachining quality could be controlled by tuning the laser processing parameters, there is undoubtedly a favorable perspective for its industrial applications. To address these issues, we develop a hybrid pulsed green laser micromachining technique to enhance the light absorption and improve the processing quality. Firstly, a sapphire surface is grooved at a low laser fluence but a high pulse overlap ratio, which leads to the absorption enhancement of the green light. Secondly, a subsequent higher laser fluence is applied at the same position. A higher ablation efficiency and better machining quality can be achieved by this hybrid laser micromachining method.

\section{Experimental}

\subsection{Laser micromachining setup}

Figure 1 shows the experimental setup of pulsed green laser micromachining of sapphire substrates. A $532 \mathrm{~nm}$ DPSS Nd:YAG laser (Lightwave, Series 210G) at a pulse duration of $45 \mathrm{~ns}$ is used as a light source. The laser beam goes through a beam attenuator to tune the laser energy, following through a beam expander to enlarge the beam diameter. The laser beam is reflected by a galvanometer, which can scan the laser beam on the target surface at a high speed by the fast rotating of the attached X \& Y mirrors. The theta-lens of the galvanometer can ensure the focal plane of the laser beam on the target surface. Its focal length is $100 \mathrm{~mm}$ and can achieve a $50 \times 50 \mathrm{~mm}$ scanning region. The galvanometer is controlled by a $\mathrm{PC}$ through the software Mark Pro. The shutter is controlled by a computer generated signal. The pulse repetition rate used in the ex- 
periment is fixed at $1 \mathrm{kHz}$, and the laser fluence varies in a range from 0 to $300 \mathrm{~J} / \mathrm{cm}^{2}$. All the experiments are carried out without any process gas assist and at a spot size of 16 $\mu \mathrm{m}$ on the substrate surface. The hybrid laser micromachining of sapphire is conducted in two steps, as listed in Table 1. For step I, the sapphire surface is scanned at a laser fluence of $25,35,50,100,150,200,250$ and $300 \mathrm{~J} / \mathrm{cm}^{2}$, respectively. For step II, a subsequent higher laser fluence of $300 \mathrm{~J} / \mathrm{cm}^{2}$ is scanned along the groove formed after step I. Scanning velocity is set at $5 \mathrm{~mm} / \mathrm{s}$. The process of the step I laser irradiation is actually the direct laser micromachining.

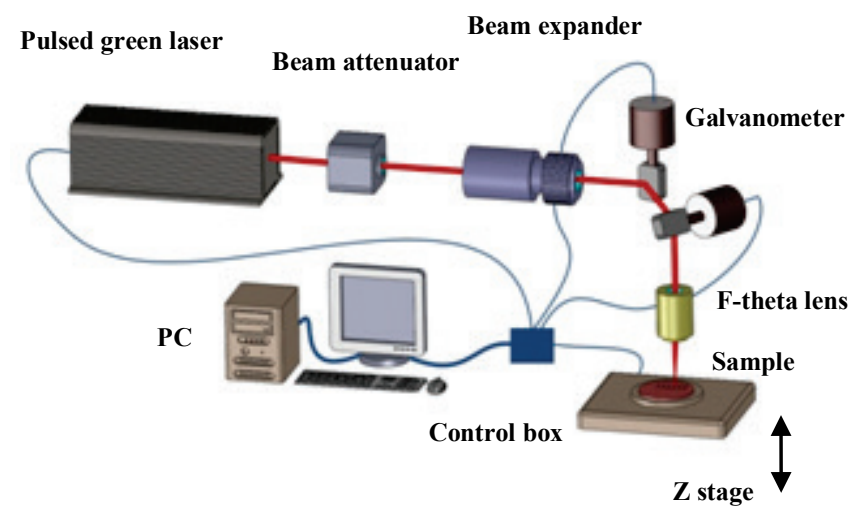

Fig. 1 Schematic diagram of the pulsed green laser micromachining of sapphire substrates.

Table 1 Hybrid laser micromachining parameters for sapphire ablation

\begin{tabular}{ccccccccc}
\hline \multicolumn{8}{c}{ Laser Fluence $\left(\mathrm{J} / \mathrm{cm}^{2}\right)$} \\
\hline Step I & 25 & 35 & 50 & 100 & 150 & 200 & 250 & 300 \\
\hline Step II & 300 & 300 & 300 & 300 & 300 & 300 & 300 & 300 \\
\hline
\end{tabular}

\subsection{Characterization}

The sapphire substrates at a dimension of $15 \times 15 \times 0.4$ $\mathrm{mm}$ are used as samples for the laser micromachining. All the samples after the laser micromachining are cleaned by 2 mol KOH solution for 5 minutes, then ultrosonic cleaned by deionized water for 5 minutes to remove debris \& contaminants. Crystallographic structure is characterized by XRD (Siemens D5005) and absorptance by UV-VIS spectrometer (UV-1800, Shimadzu). Groove depth and width are measured by a surface profilometer (Mitutoyo SV3000), while the surface morphology is observed by an optical microscope (Zxiss AX10) and scanning electron microscope (Philips Nova NanoSEM 230). All the measurement data of the groove depth and width are the average values of three readings.

\section{Results and discussion}

\subsection{Effects of laser fluence on groove depth and width}

Figure 2 shows the groove depth as a function of laser fluence for the direct and hybrid laser micromachining. Both the groove depths increase with laser fluence. This is due to more energy being absorbed and resulting in more materials removed. Figure 3 shows the groove width as a function of laser fluence for the direct and hybrid laser micromachining. The groove width increases with laser fluence as well. While the groove width for the hybrid laser micromachining varies slightly, then increases with laser fluence when the laser fluence is higher than $200 \mathrm{~J} / \mathrm{cm}^{2}$.

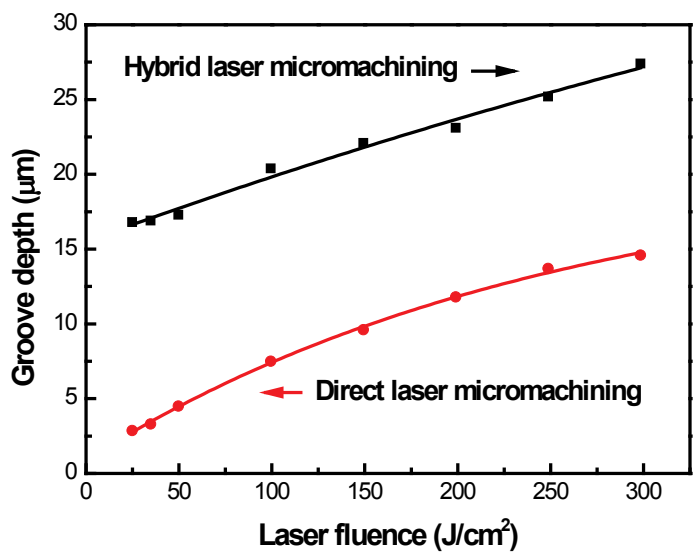

Fig. 2 Groove depth as a function of laser fluence for the direct and hybrid laser micromachining of sapphire.

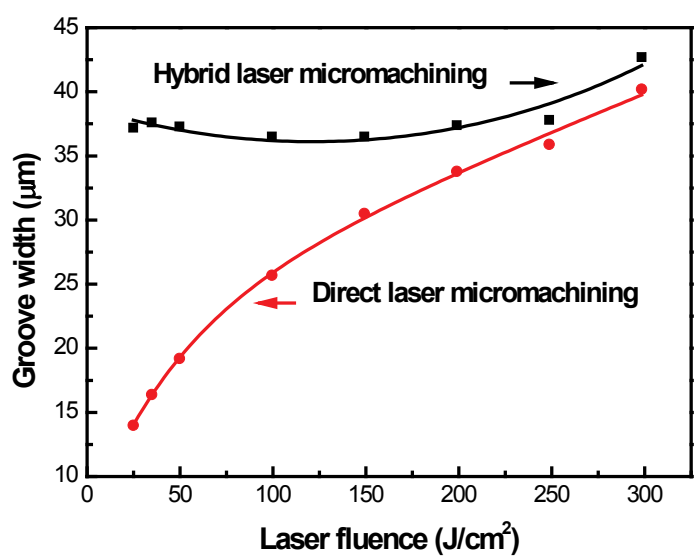

Fig. 3 Groove width as a function of laser fluence for the direct and hybrid laser micromachining of sapphire.
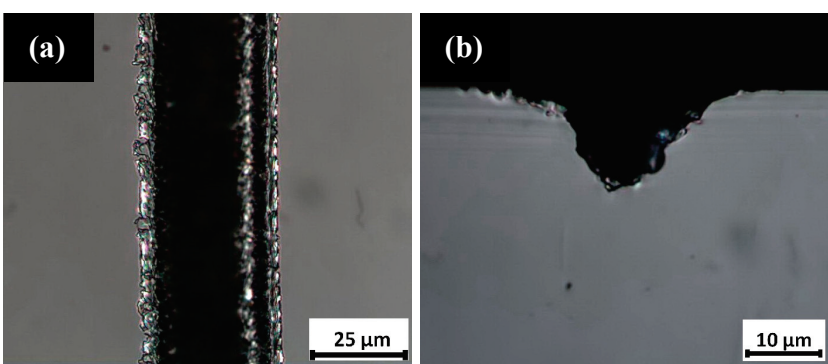

Fig. 4 Optical microscope images of the (a) front and (b) cross section views of sapphire substrates after the direct laser micromachining at a laser fluence of $300 \mathrm{~J} / \mathrm{cm}^{2}$.
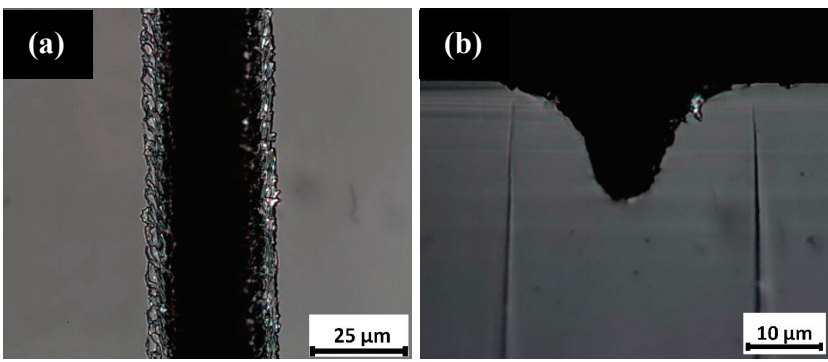

Fig. 5 Optical microscope images of the (a) front and (b) cross section views of sapphire substrates after the hybrid laser micromachining at a laser fluence of 25 (step I ) and $300 \mathrm{~J} / \mathrm{cm}^{2}$ (step II). 
Figures $4 \& 5$ show optical microscope images of the front and cross section views of sapphire substrates after the direct and hybrid laser micromachining, respectively. The groove depth of hybrid laser micromachining at a laser fluence of 25 (step I) and $300 \mathrm{~J} / \mathrm{cm}^{2}$ (step II) is higher and the groove width is lower than the direct laser micromachining at a laser fluence of $300 \mathrm{~J} / \mathrm{cm}^{2}$. This is dependent on the surface condition after the step I laser irradiation. Figure 6 shows the surface roughness increases greatly as the laser fluence is lower than 150 $\mathrm{J} / \mathrm{cm}^{2}$, and then saturates as the laser fluence increases further. The surface roughness increase results in the higher absorption after the step I laser irradiation. Therefore, the groove depth increases and groove width decreases.

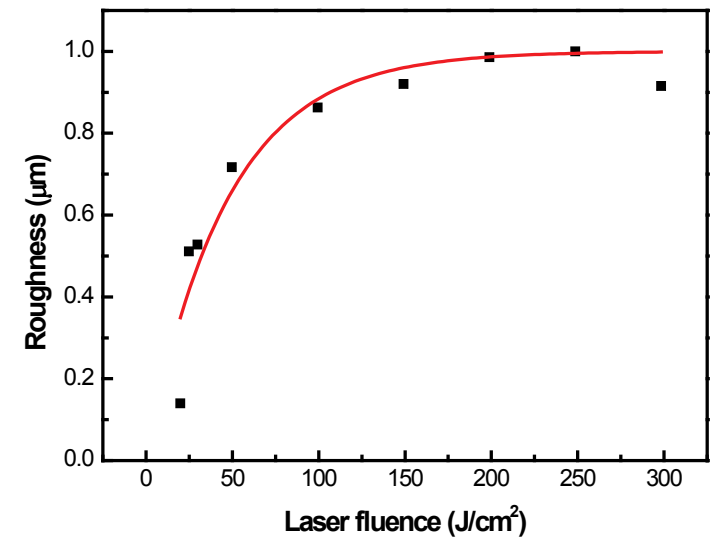

Fig. 6 Surface roughness as a function of laser fluence by the pulsed green laser ablation of sapphire.

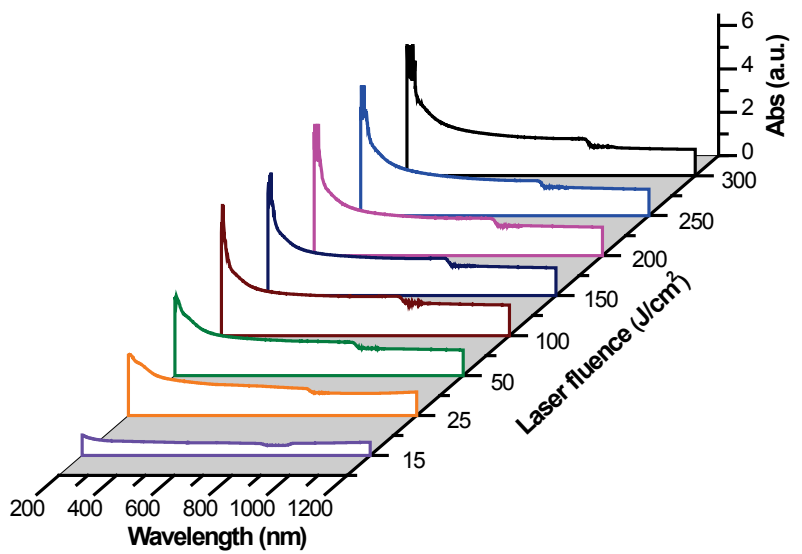

Fig. 7 UV-VIS absorption spectra of sapphire after the step I laser irradiation at different laser fluences.

UV-VIS absorption spectra of sapphire at different laser fluences are shown in Fig. 7. At a wavelength $<300 \mathrm{~nm}$, there is a high peak absorptance for the sapphire. While the absorptance is very low from 300 to $1200 \mathrm{~nm}$, particularly in IR light. This means deep UV light is superior for the sapphire absorption. Extracted data from Fig. 7 is the absorptance as a function of laser fluence at a wavelength of $532 \mathrm{~nm}$, as shown in Fig. 8. Original sapphire's absorptance is near zero and almost transparent to $532 \mathrm{~nm}$ laser irradiation. When the laser fluence is below $15 \mathrm{~J} / \mathrm{cm}^{2}$, no laser ablation occurs and sapphire crystallographic structure is in alpha phase with two peaks observed at theta de- grees of 25.5 and 52.5. The absorptance starts to increases sharply when the laser fluence is higher than $15 \mathrm{~J} / \mathrm{cm}^{2}$. Then it increases when the laser fluence is up to $35 \mathrm{~J} / \mathrm{cm}^{2}$. At this stage, the sapphire surface is changed from crystallite to amorphous, as shown in Fig. 9. If the laser fluence continues to increase up to $50 \mathrm{~J} / \mathrm{cm}^{2}$, the light absorptance saturates. In our further experiment, the laser fluence is set at lower than $50 \mathrm{~J} / \mathrm{cm}^{2}$.

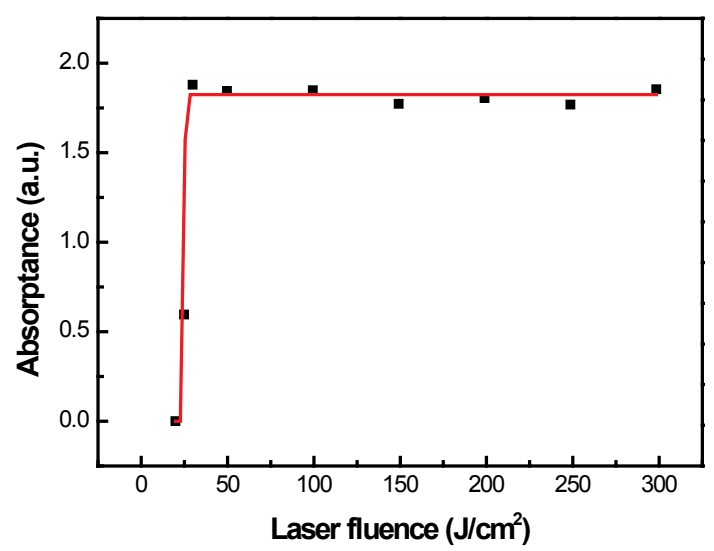

Fig. 8 Light absorptance as a function of laser fluence at a wavelength of $532 \mathrm{~nm}$.

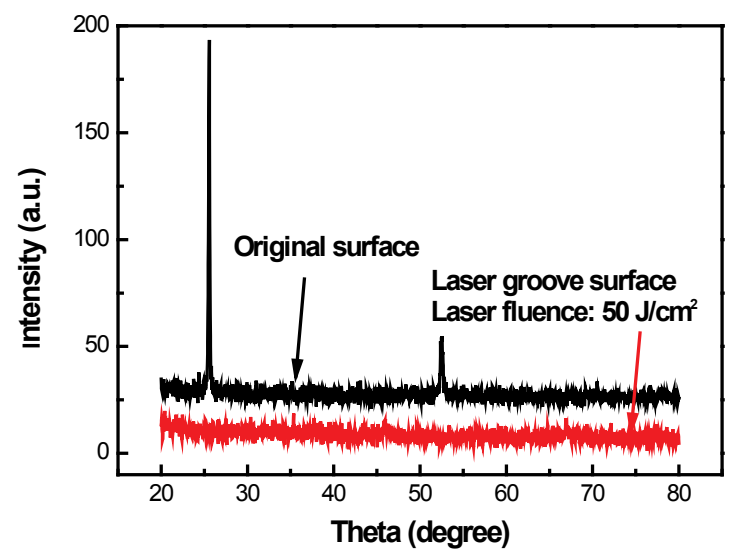

Fig. 9 XRD analyses of the pulsed green laser ablated surface (intensity of the laser ablated surface is displaced 20 above the original surface for easy comparison).

\subsection{Morphology change}

\subsubsection{Formation of redeposited debris}

Laser in the experiment ( $\lambda=532 \mathrm{~nm}, \mathrm{tp}=45 \mathrm{~ns})$ is used to micromachining the sapphire without any reactive gas or chemical solution assist. Photon energy of the green laser light is approximately $2.6 \mathrm{eV}$, which is much lower than the bandgap of sapphire at $8.0 \mathrm{eV}$. Laser excitation cannot result in direct bond breaking. Therefore, the nanosecond green laser ablation of sapphire has been analyzed on the basis of photo-thermal process. The photo-thermal ablation is based on the excitation energy being instantaneously transformed into heat. Due to the rapid dissipation of the excitation energy from the electrons to the lattice, the material surface is heated rapidly and vaporized explosively with surface melting. It is found that the laser scanning creates a certain amount of the redeposited debris in the ablation crater. The size of redeposited debris is approximately several micrometers in thickness and width. The higher the laser fluence is, the larger the redeposited debris 
is. The formation of redeposited debris enlarges the groove width. Chemical composition of redeposited debris is the same as sapphire substrate $\left(\mathrm{Al}_{2} \mathrm{O}_{3}\right)$. It is hardly removed after chemical cleaning because of its high hardness and resistant to chemical etching. Because the light absorption is enhanced for the hybrid laser micromachining, the redeposited debris adhered to the groove is shallower than the direct laser micromachining.

\subsubsection{Thermal stress induced micro-cracks}

Enlarged images of the redeposited debris and groove bottom areas show the formation of micro-cracks for the hybrid laser micromachining. This is due to thermal stress caused when the sapphire surface's rapidly heating and cooling to room temperature creates a high thermal gradient. The sapphire substrate is under compression at the early laser heating stage and under tension at the later laser cooling stage. A release wave is initiated at the surface or due to a decaying compressional wave. Interaction of the two opposing release waves induces tensile stresses and cracks. The thermal stress $(\sigma)$ can be estimated by $[16,17]$ :

$$
o=G \gamma \frac{2 A I \sqrt{\alpha t}}{\kappa(1-v)} \operatorname{ierfc}\left(\frac{z}{\sqrt{4 \alpha t}}\right),
$$

where $G$ demotes Young's modulus, $\gamma$ the linear thermal expansion coefficient, $A$ the absorption coefficient, $I$ the laser fluence, $\alpha$ the thermal diffusivity, $t$ the heating time, $\kappa$ the thermal conductivity, $\mathrm{z}$ the distance from the surface and $v$ the poisons' ratio. From equation (1), a higher laser fluence results in a higher thermal stress.

\subsubsection{Reducing redeposited debris and micro-cracks}

Although the hybrid laser micromachining can achieve a higher ablation quality than the direct laser micromachining in aspects of groove depth and width, the microcracks of sapphire surface are hardly avoided due to a higher laser fluence applied at the step II laser irradiation. By setting sapphire's thermal physical properties [18] such as Young's modulus $\mathrm{G}=3.45 \times 10^{11} \mathrm{~Pa}$, thermal expansion coefficient $\gamma=4.3 \times 10^{-6} \mathrm{~K}^{-1}$, thermal conductivity $\kappa=0.325 \mathrm{~W} / \mathrm{cm} \cdot \mathrm{K}$, absorption coefficient $\mathrm{A}=0.03$, thermal diffusivity $\alpha=0.096 \mathrm{~cm}^{2} / \mathrm{s}$, heating time $\mathrm{t}=45 \mathrm{~ns}$, poisson's ratio $v=0.18$, and fracture strength $\sigma_{\text {th }}=20 \mathrm{GPa}$, the threshold laser fluence for the crack formation can be calculated as $\mathrm{I}_{\mathrm{th}}=75 \mathrm{~J} / \mathrm{cm}^{2}$. Therefore, the micro-cracks occur when the laser fluence is $300 \mathrm{~J} / \mathrm{cm}^{2}$ at the step II laser irradiation. When the laser fluence is below $75 \mathrm{~J} / \mathrm{cm}^{2}$, the micro-cracks may be minimized or even avoided. Based on the analysis, a lower laser fluence for the step II laser irradiation is applied to reduce the redeposited debris and micro-cracks. Meanwhile, multiple scans should be applied to increase the groove depth. A lower subsequent laser fluence of $50 \mathrm{~J} / \mathrm{cm}^{2}$ for 2 scan passes are used to process the sapphire, as shown in Fig. 10. It shows the redeposited debris and micro-cracks are remarkably reduced, but there still exists some redeposited debris above the groove. If a subsequent laser fluence decreases to 25 $\mathrm{J} / \mathrm{cm}^{2}$ for 5 scan passes, a clean groove can be achieved, as shown in Fig. 11. Based on the above analysis, we use a $25 \mathrm{~J} / \mathrm{cm}^{2}$ (step I) and $25 \mathrm{~J} / \mathrm{cm}^{2}$ (step II) for 5 scan passes to fabricate microstructures on the sapphire substrates, as shown in Fig. 12. It shows a high processing quality mi- crostructures are achieved by the hybrid laser micromachining. This means the hybrid laser micromachining at a lower laser fluence for the step II laser irradiation and multiple scans is an effective approach for a high quality micromachining of transparent sapphire.

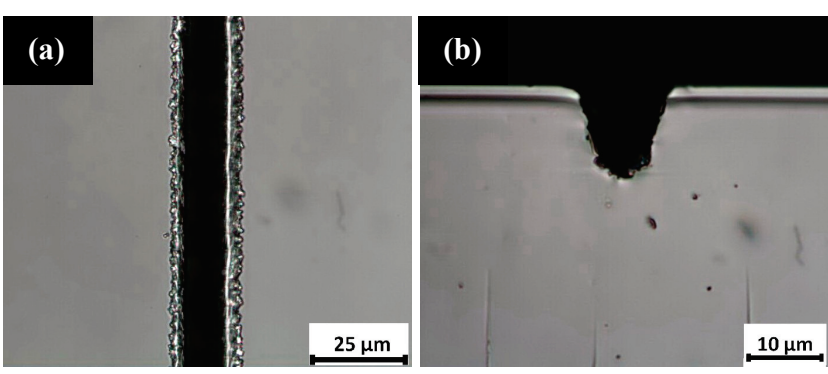

Fig. 10 Optical microscope images of the (a) front and (b) cross section views for the hybrid laser micromachining sapphire at a laser fluence of $25 \mathrm{~J} / \mathrm{cm}^{2}$ (step I) and $50 \mathrm{~J} / \mathrm{cm}^{2}$ (step II) for 2 scans.
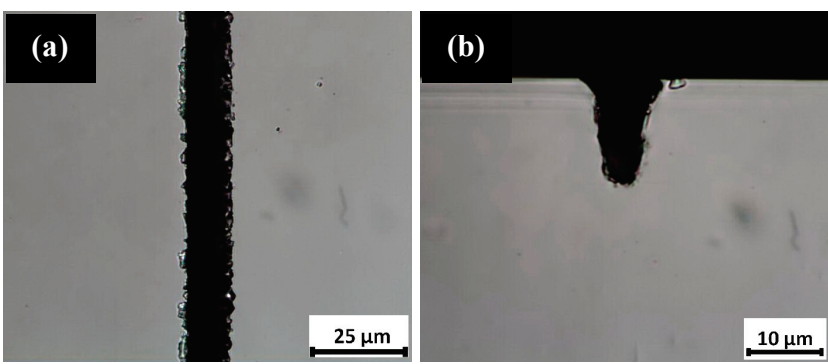

Fig. 11 Optical microscope images of the (a) front and (b) cross section views for the hybrid laser micromachining sapphire at a laser fluence of $25 \mathrm{~J} / \mathrm{cm}^{2}$ (step I ) and $25 \mathrm{~J} / \mathrm{cm}^{2}$ (step II) for 5 scans.
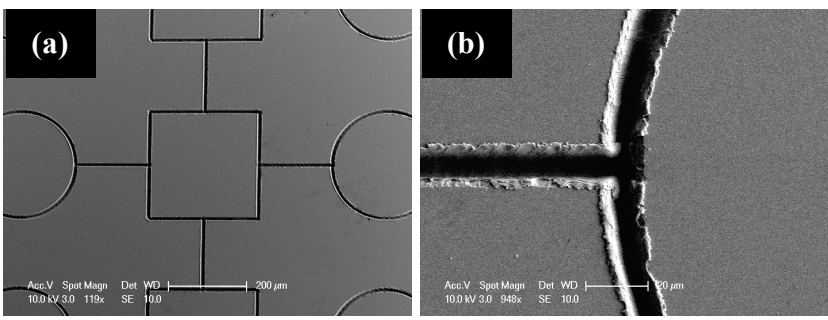

Fig. 12 SEM images of microstructure made on sapphire substrates by the hybrid laser micromachining at different magnification scales. Scale bars in (a) and (b) are 200 and $20 \mu \mathrm{m}$ respectively.

\section{Conclusions}

In this paper, we develop a new hybrid pulsed green laser micromachining technique to enhance the energy absorption efficiency on the sapphire substrate and achieve a high quality laser micromachining of this functional but difficultly processed materials. It is found that both the groove depths increase with laser fluence for the direct \& hybrid laser micromachining. The groove width also increases with laser fluence at the step I laser irradiation. While it varies slightly at the step II laser irradiation as the laser fluence is lower than $200 \mathrm{~J} / \mathrm{cm}^{2}$, then increases with laser fluence. Meanwhile, sapphire structure is modified from crystallite to amorphous state after the laser irradiation. Both the light absorption and surface roughness after 
the step I laser irradiation increase sharply and saturate with laser fluence. Hybrid laser micromachining of the transparent sapphire can achieve much higher quality than the direct laser micromachining in aspects of deeper groove depth and narrower groove width. In order to reduce redeposited debris and micro-cracks, a hybrid laser micromachining method at a lower laser fluence for the step II laser irradiation and multiple scans can achieve a high quality processing of the substrate.

\section{Acknowledgments}

The research is supported by National Nature Science Foundation of China (No.50805027 and 50675038).

\section{References}

[1] A. Salleo, T. Sands and F.Y. G'enin: Applied Physics A, 71, (2000) 601 .

[2] Z.Q. Huang, M.H. Hong, T.B.M and Q.Y. Lin: Applied Physics A, 93, (2008) 159.

[3] Bin Lan, Ming-Hui Hong, Kai-Dong Ye, Zeng-Bo Wang, Shi-Xin Cheng and Tow-Chong Chong: Jpn. J. Appl. Phys. 43, (2004) 7102.

[4] Joseph R. Bashe, Gene Dempsey, Ikerionwu A. Akwani, Keith T. Jacoby and Douglas L. Hibbard: Proc. SPIE, Window and Dome Technologies and Materials X, Orlando, (2007) p.654517.

[5] Muttamara Apiwat, Fukuzawa Yasushi, Mohri Naotake and Tani Takayuki: Materials Transactions, 45, (2004) 2486.

[6] Shengjun Zhoua and Sheng Liu: Applied Surface Science, 255, (2009) 9469.

[7] Ximing Ding, Tadatake Sato, Yoshizo Kawasachi and Hiroyuki Niino: Jpn. J. Appl. Phys., 42, (2003) L176.

[8] D. Karnakisa, E.K. Illy, M.R.H. Knowles, E. Gu and M.D. Dawson: Light-Emitting Diodes: Research Manufacturing and Applications VIII, San Jose, (2004) p.207.

[9] Hwee Ming Lam, MingHui Hong, Shu Yuan and Tow Chong Chong: Proceedings of SPIE, Third International Symposium on Laser Precision Microfabrication, Osaka, (2003) p.114

[10] D. Ashkenasi, A. Rosenfeld, H. Varel, M. Wähmer and E.E.B. Campbell: Applied Surface Science, 120, (1997) 65

[11] Vygantas Mizeikis, Shigeru Kimura, Nikolay V, Vygandas Jarutis, Akira Saito, Hiroaki Misawa and Saulius Juodkazis: Applied Surface Science, 255, (2009) 9745.

[12] Hideyuki Horisawa, Hajime Emura and Nobuo Yasunaga: Vacuum, 73, (2004) 661.

[13]E.K. Illy, M. Knowles, E. Gu and M.D. Dawson: Applied Surface Science, 249, (2005) 354.

[14] Tai-Chang Chen and Robert B. Darling: Journal of Materials Processing Technology, 169, (2005) 214.
[15]Xie Xiao-zhu, Huang Fu-min, Wei Xin, Hu Wei and Ren Qing-lei: Proceedings of SPIE, Semiconductor Lasers and Applications IV, Beijing, (2010) p.78440T.

[16] H. S. Carslaw and J. C. Jaeger: "Conduction of Heat in Solids" (second edition), (Publisher, Oxford University Press, 1959).

[17]Bruno A Boley and Jerome H Weiner: "Theory of Thermal Stresses", (Publisher, Dover Publications, 1997).

[18] Dobrovinskaya Elena R., Lytvynov Leonid A. and Pishchik Valerian: "Sapphire: Material, Manufacturing, Applications", (Publisher, Springer, 2009).

(Received: June 02, 2011, Accepted: November 09, 2011) 